\title{
Eye movements are functional during face learning
}

\author{
JOHN M. HENDERSON, CARRICK C. WILLIAMS, and RICHARD J. FALK \\ Michigan State University, East Lansing, Michigan
}

\begin{abstract}
In a free viewing learning condition, participants were allowed to move their eyes naturally as they learned a set of new faces. In a restricted viewing learning condition, participants remained fixated in a single central location as they learned the new faces. Recognition of the learned faces was then tested following the two learning conditions. Eye movements were recorded during the free viewing learning condition, as well as during recognition. The recognition results showed a clear deficit following the restricted viewing condition, compared with the free viewing condition, demonstrating that eye movements play a functional role during human face learning. Furthermore, the features selected for fixation during recognition were similar following free viewing and restricted viewing learning, suggesting that the eye movements generated during recognition are not simply a recapitulation of those produced during learning.
\end{abstract}

The active control of eye fixation plays an important functional role in a wide variety of cognitive and perceptual tasks. For example, fixation sites and fixation durations are closely time-locked to ongoing perceptual and cognitive processes during reading and picture perception (Buswell, 1935; Tinker, 1946; Yarbus, 1967; for recent reviews, see Henderson, 2003; Henderson \& Ferreira, 2004; Rayner, 1998). A similar real-time coupling between eye movements and ongoing perceptual and cognitive processing has been shown in many complex perceptual, cognitive, and motor tasks (e.g., Cutting, Alliprandini, \& Wang, 2000; Hayhoe, Shrivastava, Mruczek, \& Pelz, 2003; Land, Mennie, \& Rusted, 1999; Tanenhaus, Spivey-Knowlton, Eberhard, \& Sedivy, 1995).

In the present study, we investigated human eye movements in the biologically and psychologically important task of face learning. Faces are arguably the most important and salient visual stimulus a human encounters. Faces are central in human social interaction, providing critical information about the age, gender, emotional state, intention, and identity of another. Behavioral, developmental, neuropsychological, and neuroimaging studies provide evidence that face perception is supported by dedicated computational and neural processing systems (e.g., Farah, Wilson, Drain, \& Tanaka, 1998;

This research was supported by National Science Foundation Grants BCS-0094433 and ECS-9874541 and Army Research Office Grant DAAD19-00-1-0519. The opinions expressed in this article are those of the authors and do not necessarily represent the views of the Department of the Army or any other governmental organization. Reference to or citations of trade or corporate names does not constitute explicit or implied endorsement of those entities or their products by the authors or the Department of the Army. We thank Keith Rayner and Geoff Loftus for their helpful comments. Correspondence should be addressed to J. M. Henderson, Psychology Building, Michigan State University, East Lansing, MI 48824-1116 (e-mail: john@eyelab.msu.edu).
Goren, Sarty, \& Wu, 1975; Kanwisher, McDermott, \& Chun, 1997; McCarthy, Puce, Gore, \& Allison, 1997; but see Gauthier, Tarr, Anderson, Skudlarski, \& Gore, 1999, for a different interpretation). The majority of research on eye movements during face perception has focused on face recognition (e.g., Althoff \& Cohen, 1999; Luria \& Strauss, 1978; Walker-Smith, Gale, \& Findlay, 1977). In contrast, little is known about eye movements during face learning. For example, it has yet to be determined whether eye movements are actually functional in the learning process.

Despite the importance of eye movements in many visual-cognitive tasks, it is not clear that they must play a functional role in face learning, because faces are thought to be processed holistically (for a review see Tanaka \& Farah, 2003). This holistic processing has been proposed to underlie the face-specific effects observed in behavioral studies of the face inversion effect (Yin, 1969), the double-dissociation between face recognition and object recognition observed in lesion studies (Newcombe, Mehta, \& de Haan, 1994), and the specificity of the fusiform face area of temporal cortex observed in functional neuroimaging experiments (Kanwisher et al., 1997). Holistic processing is thought to be based on relational information among parts of a face (Tanaka \& Farah, 2003). Such relational information could be derived from low spatial frequency analysis that does not require foveal vision, in much the same way that scenes can be recognized as a member of a particular class on the basis of scene statistics related to low spatial frequency information (Oliva \& Schyns, 2000). That is, to the extent that faces are encoded holistically, fixation of specific facial features may not be necessary for learning. According to this hypothesis, any eye movements observed during face learning serve some other function, such as encoding subidentity detail or emotion. 
In the present study, participants were asked to learn unfamiliar faces in preparation for a later memory test. The faces were presented in two blocked conditions. In the free viewing condition, participants were free to move their eyes as they chose. In the restricted viewing condition, participants were required to keep their eyes fixated in one central position. The faces were visible for the same total amount of viewing time in the two learning conditions. Following the learning session, a recognition session was given in which the previously learned faces were mixed with similar new faces and participants were asked to distinguish the old from the new. If eye movements play a functional role in face learning, recognition performance should be better in the free viewing than in the restricted viewing condition. If, instead, eye movements do not play a functional role in face learning, recognition should be equivalent in the two conditions.

We examined several additional questions. First, to what extent are the features selected for fixation similar in face learning and recognition? One reason to consider the similarity in eye movements during learning and recognition arises from recent neuropsychological data suggesting that the systems supporting face learning and recognition can be dissociated (Kanwisher \& Moscovitch, 2000; Tippet, Miller, \& Farah, 2000). If face learning and face recognition are supported by separate computational and neural systems with their own information-gathering needs, eye movements during learning and recognition might differ from each other. On the other hand, to the extent that face perception is accomplished by a common domain-specific module (e.g., Dubois et al., 1999; Gorno Tempini et al., 1998), very similar eye-movement behavior would be expected during learning and recognition.

Second, we examined the nature of the features selected for fixation during learning and recognition, and for learned and unlearned faces during recognition. In an important study, Yarbus (1967) presented a pictorial example of a viewer's eye-movement pattern over the profile bust of Nefertiti, the ancient Egyptian queen. The viewing pattern suggested that a large percentage of viewing time was spent on the outside bounding contours of the face. In the behavioral literature, there is some suggestion that the external features of a face are more important to recognition when the face is unfamiliar than when it is familiar (Ellis, Shepherd, \& Davies, 1979; Young, Hay, McWeeny, Flude, \& Ellis, 1985). On the other hand, there is considerable evidence from eye-movement studies that fixation is directed predominantly to internal facial features during recognition. For example, Janik, Wellens, Goldberg, and Dell'Osso (1978) found that when viewers were asked to form an impression of faces presented for $15 \mathrm{sec}$ each, $43.4 \%$ of the fixation time was spent on the eyes. Henderson, Falk, Minut, Dyer, and Mahadevan (2001) reported that closer to $60 \%$ of fixation time was spent on the eyes in a face-recognition task and that about $90 \%$ of fixation time was spent on the eyes, nose, and mouth. Walker-Smith et al. (1977) examined the viewing strategies of 3 participants in a delayedrecognition task and a task in which two faces were to be simultaneously compared and also found that fixations tended to be primarily restricted to the eyes, nose, and mouth. Groner, Walder, and Groner (1984) reported that the largest proportion of three-region transitions during face perception involved scanning between the eyes. Strong biases toward fixating internal facial features have also been reported by Althoff and Cohen (1999), Luria and Strauss (1978), and Mertens, Siegmund, and Grüsser (1993). The present study provided an opportunity to examine whether fixations on the external and internal features of faces differ during learning and recognition and for old versus new faces during recognition.

Finally, according to the scan path theory of perceptual recognition (Noton \& Stark, 1971a, 1971b; Stark \& Ellis, 1981), the representation of a complex pattern includes the perceptuo-motor cycle used to encode it. In this view, eye movements generated during visual learning must be recapitulated during recognition for recognition to be successful. The observation of some similarity in fixation patterns across learning and recognition was originally taken to support the scan path theory (Noton \& Stark, 1971a, 1971b), and recent studies have extended the idea of scan paths to other domains such as visual imagery (Brant \& Stark, 1997). However, for the most part the evidence has not supported the scan path theory (Henderson, 2003; Rayner, 1998). For example, it is clear that the complex visual stimuli that typically elicit eye movements can be recognized in tachistoscopic presentations in which eye movements are not possible (e.g., Potter, 1976; Schyns \& Oliva, 1994; Thorpe, Fize, \& Marlot, 1996). Here we tested a complementary and previously untested prediction of the scan path theory: If the eye movements that are encoded during learning must be recapitulated during recognition, and if eye movements are prevented during learning, participants should refrain from making eye movements during recognition. A weaker prediction is that if participants do make eye movements during recognition following static learning, such movements should differ from those generated during recognition following free movement learning because only in the latter condition can the recognition movements recapitulate those generated during learning.

\section{METHOD}

Participants viewed 20 faces for $10 \mathrm{sec}$ each during a learning session and then were tested on those faces and 20 new faces in a recognition session. Two learning conditions were compared: In the free viewing learning condition, participants were allowed to move their eyes freely during learning. In the restricted viewing learning condition, participants were required to maintain fixation in the center of each face during learning, and any deviation from that position caused the display to be replaced by a mask. Scores in the recognition session were compared as a function of learning condition. In addition, eye movement patterns were compared from learning to recognition, and during recognition as a function of whether eye movements were free or restricted during learning. 


\section{Participants}

Eight members of the Michigan State University undergraduate participant pool participated in this experiment. All had normal, uncorrected vision and received course credit for participation.

\section{Stimuli}

Digitized images of the faces of 40 Michigan State University female undergraduate students, all approximately the same age and of the same ethnicity, served as stimuli. The images were obtained with a digital camera under similar lighting conditions and with a similar neutral expression. The images were imported into Adobe Photoshop, differences in luminance and contrast were equalized, and hair was edited to a uniform style. None of the faces had any overly distinguishing characteristics such as jewelry or idiosyncratic makeup. The resulting images were $400 \times 550$ pixels in size and subtended $7.84^{\circ}$ of visual angle horizontally and $10.78^{\circ}$ vertically at a viewing distance of $1 \mathrm{~m}$, approximately equivalent to the size of a real face (approximately $14 \mathrm{~cm}$ wide) viewed from a distance of $1 \mathrm{~m}$. The faces were embedded in an $800 \times 600$ pixel gray background for presentation. Figure 1 displays an example face used in the experiment.

\section{Apparatus}

The stimuli were displayed at a resolution of $800 \times 600$ pixels $\times$ 32,768 colors on an NEC Multisync XE 15 -in. monitor driven by a Hercules Dynamite Pro super video graphics adapter (SVGA) card. Eye movements were monitored with a Generation 5.5 Stanford Research Institute Dual Purkinje Image Eye Tracker (Crane, 1994), which has a resolution of 1 arc min and a linear output over the range of the visual display used. A bite-bar and forehead rest maintained the participant's viewing position and distance. The right eye was tracked, although viewing was binocular. Signals were sampled from the eye tracker with the polling mode of the Date Translations DT2803 analog-to-digital converter, producing a sampling rate slightly greater than $1,000 \mathrm{~Hz}$.

\section{Procedure}

The participants first read a description of the experiment along with a set of instructions. A bite-bar was prepared, the experimental task was explained, and questions were answered. The participants were informed that there were two parts to the experiment, a learning session and a recognition session, and that the learning session would be divided into free viewing and restricted viewing blocks. The order of presentation of these blocks was counterbal-

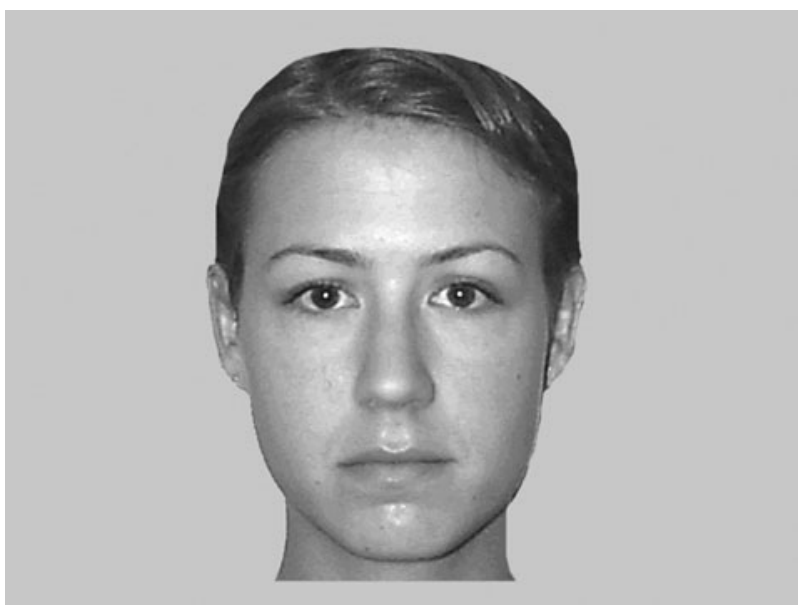

Figure 1. Example face used in the experiment. Faces were presented in color. anced across participants. In the free viewing block, the participants were told that they were free to move their eyes over faces as they wished. In the restricted viewing block, the participants were instructed to keep their gaze on the center of the faces. The center of each face was defined as the area directly between the eyes.

The center of each face was marked with a red square and cross subtending $1^{\circ}$ of visual angle. In the restricted viewing condition, the participants were informed that the face would disappear from view if their gaze left the confines of the red square; if the participant's gaze did leave the square, the face disappeared and was replaced with a multicolored mask containing the red square. The participants had to return their gaze to this square before the face would reappear. This procedure ensured that the participant could not execute eye movements in the service of the learning task.

In both the free and restricted viewing conditions, the participants were instructed to study the faces as carefully as possible to prepare for the memory test. The nature and difficulty of the test was explained. Following presentation of the 10 faces that were studied in each block, five full-color photographs depicting natural scenes were presented for study. The participants were told to memorize the scenes to prepare for a test that would be given at the end of the experiment. The scenes were included as interpolated filler material to provide a delay between the study and the recognition sessions for the faces. The memory test for the scenes was not given, however.

To calibrate the eye tracker, the participants fixated a series of markers presented on the display monitor. Calibration was checked by displaying a fixation marker indicating the computer's estimate of the current fixation position. Calibration was considered accurate when the estimated fixation position was within \pm 5 arc min of each of five displayed test locations and was checked periodically throughout the experiment. The participant was recalibrated whenever this criterion was not met.

In the learning session, the experimenter initiated a trial once the participant had fixated a marker centered on the display. A trial consisted of the presentation of a face in the center of the monitor for $10 \mathrm{sec}$. In the free viewing learning condition, the face remained on the monitor continuously. In the restricted viewing condition, the face was visible only when fixation was inside the restricted viewing square; any eye position samples detected outside the square caused the face to be replaced by a mask and the presentation clock to stop. This ensured that the face was visible for $10 \mathrm{sec}$ in both the free viewing and restricted viewing conditions. Before the beginning of the restricted viewing block, the participants were given practice keeping their eyes in the center of the display, which they found easy to achieve.

In the recognition session, 40 faces ( 20 old and 20 new) were presented in a pseudorandom order. The participants were allowed to move their eyes freely during the entire recognition session. Half of the familiar faces had been studied in the free viewing condition and half in the restricted viewing condition. Thus, there were 40 test trials: 10 (items) $\times 2$ (viewing condition) $\times 2$ (old $/$ new $)$. The faces were counterbalanced across conditions via a Latin-square design so that all faces appeared in each condition an equal number of times across participants. The participants were told that when a face appeared on the monitor, they were to decide as quickly and as accurately as possible whether it had been presented in the learning session. The participants responded by using two buttons (old or new) on a button box. Each test trial began with the participant fixating a marker in the center of the screen. The face was then presented and remained in view until response. The entire experiment lasted approximately $35 \mathrm{~min}$.

\section{RESULTS}

If comparison across the free viewing and restricted viewing conditions is to be meaningful, it is important 


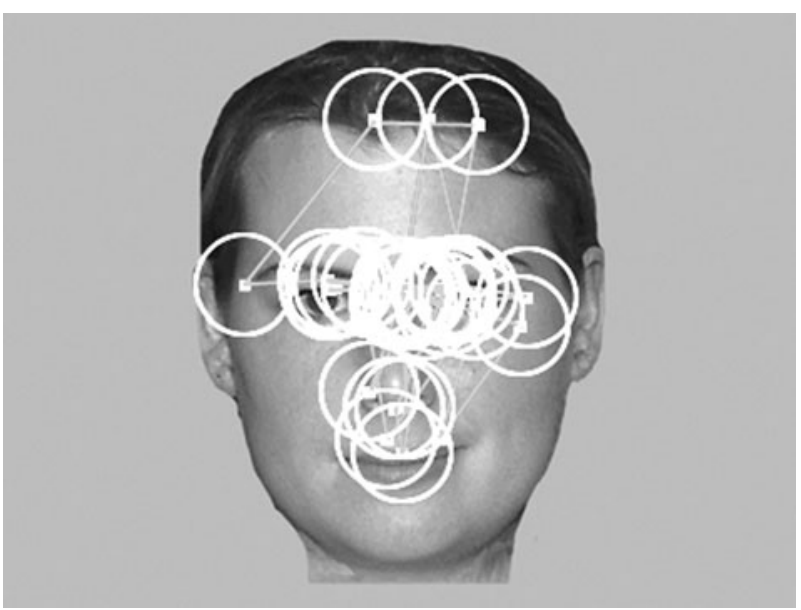

Figure 2. Example eye-movement pattern generated by one participant during the learning session. Straight lines represent saccades, and circled dots represent fixations.

first to establish that participants did, in fact, spontaneously make eye movements in the free viewing condition of the learning session. Inspection of the data indicated that all participants spontaneously moved their gaze over all the presented faces (i.e., $100 \%$ of the trials) during free viewing learning. Figure 2 shows a typical scan pattern of fixations and saccades made on a single face by a single participant during this session. Mean fixation duration was $318 \mathrm{msec}$ and mean saccade length was $2.09^{\circ}$ in the free viewing learning session.
To quantify the dispersion of eye fixations observed over the faces during face learning, each face was divided into seven nonoverlapping regions corresponding to the principal facial features (see Henderson et al., 2001; Minut, Mahadevan, Henderson, \& Dyer, 2000). These features corresponded to (1) eyes, (2) nose, (3) mouth, (4) ears, (5) chin and neck, (6) cheeks, and (7) forehead and top of head. Figure 3 shows the mean proportion of trials in which each of these regions was fixated at least once per face, collapsed across faces and participants. As can be seen in Figure 3, every participant fixated the eyes, and most participants fixated the nose and mouth of every face. The heavy emphasis on these features is similar to the data reported for fixation distributions during face recognition (Groner et al., 1984; Henderson et al., 2001; Janik et al., 1978; Walker-Smith et al., 1977). The forehead also received a relatively high proportion of fixations, perhaps reflecting the fact that hair and hairline are typically strong cues to face identity.

We also examined the total amount of time each feature was fixated in the free viewing condition of the learning phase. As shown in Figure 4, participants devoted the majority of fixation time to the eyes and much less time to the other features of the face. Participants spent just over $4 \mathrm{sec}$ of the $10 \mathrm{sec}$ of learning time for each face examining the eyes, whereas each of the other features was examined for $1 \mathrm{sec}$ or less. This analysis once again shows the dominance of the eyes as an important feature for face learning.

In summary, participants spontaneously moved their eyes during the free viewing face learning session, and

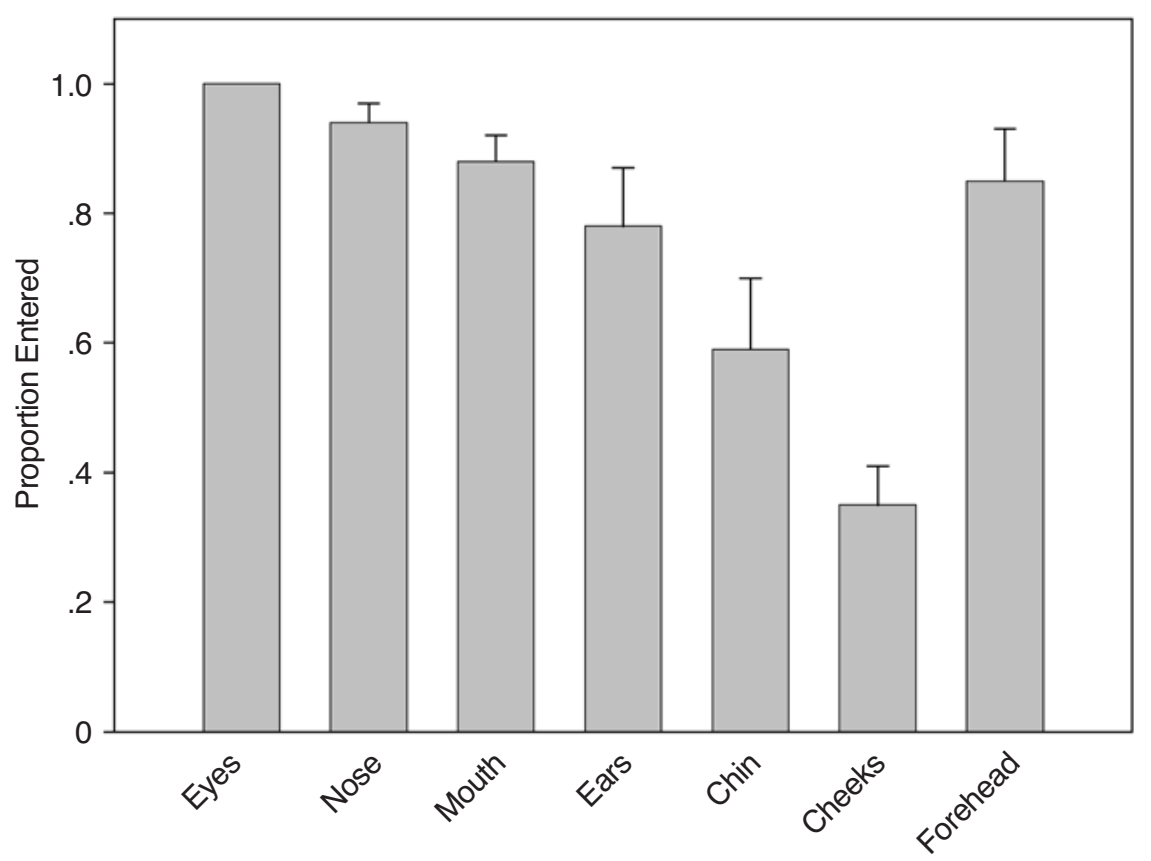

Figure 3. Mean proportion of trials in which each face region was fixated at least once during free viewing learning. Error bars represent standard errors. 


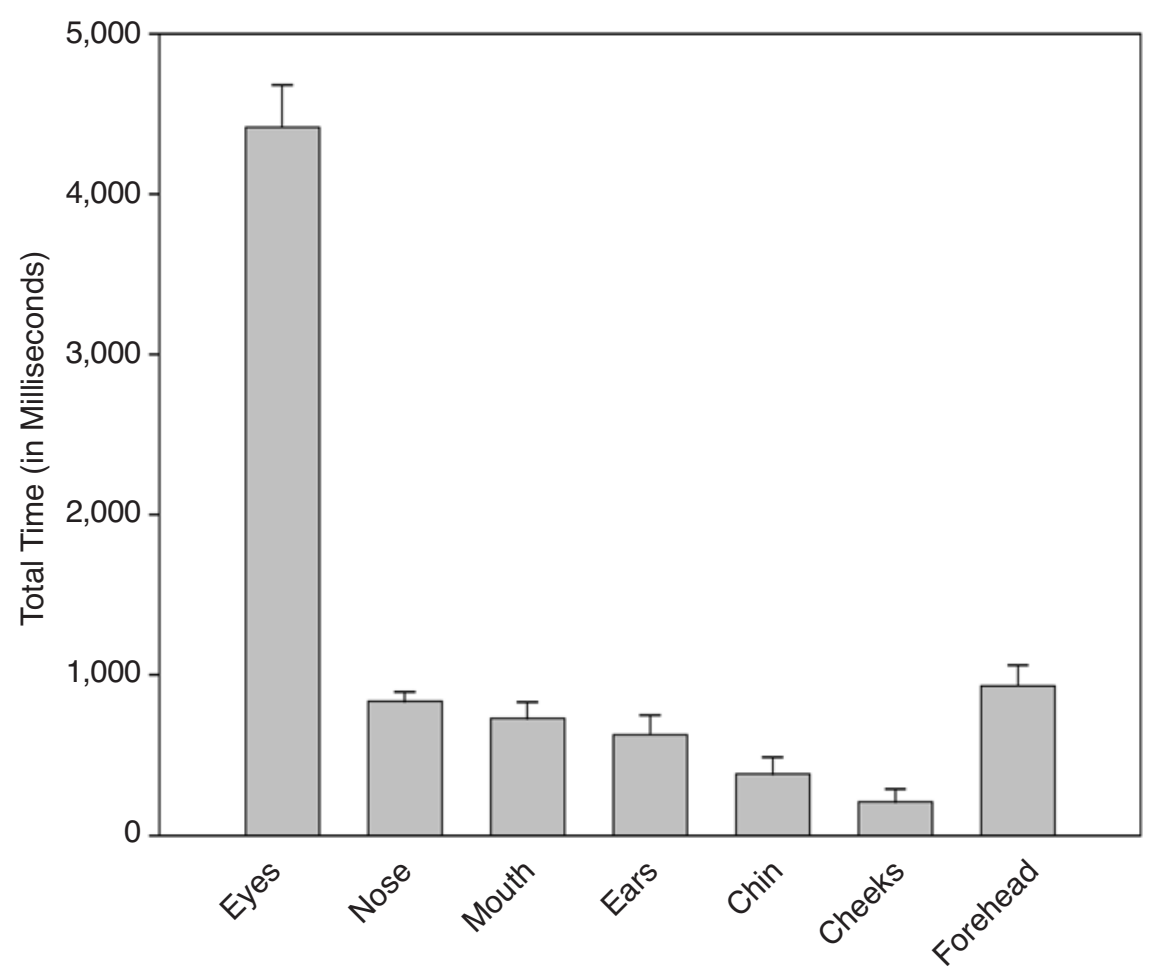

Figure 4. Average total fixation time spent in each region during free viewing learning. Error bars represent standard errors.

they were systematic in selecting specific facial features as they attempted to learn the faces. Comparison of the effect of free and restricted viewing face learning on recognition is therefore meaningful.

\section{Do eye movements facilitate face learning?}

The central question in the present study was whether eye movements are functional in face learning. If so, performance in the recognition session should be better for faces learned in the free viewing condition than in the restricted viewing condition. On the other hand, if eye movements serve some other purpose unrelated to learning (e.g., supporting the perception of emotion) or are epiphenomenal, restricting eye movements during learning should not negatively affect performance on the following recognition task. To examine these contrasting predictions, we analyzed performance in the recognition session as a function of eye movement condition in the learning session (see Table 1). Overall mean percentage correct was reliably lower for the restricted viewing condition $(52.5 \%)$ than for the free viewing condition $[81.3 \%$; $\left.F(1,7)=35.95, M S_{\mathrm{e}}=.0092, p<.001\right]$. Because only a single test session was administered, the same false alarm rate could be used for the two learning conditions. This false alarm rate was $11.9 \%$. Using $\mathrm{A}^{\prime}$, a nonparametric signal detection measure of sensitivity, we found that both conditions reliably differed from chance [free viewing $=0.912, t(7)=27.85, p<.001$; restricted viewing $=$ $.801, t(7)=8.75, p<.001]$ and from each other $\left[F(1,7)=21.41, M S_{\mathrm{e}}=.0023, p<.01\right]$. Mean response times for the restricted viewing condition $(2,078 \mathrm{msec})$ and the free viewing condition $(2,166 \mathrm{msec})$ were similar $(F<1)$. Thus, while face learning without eye movements was possible in this experiment, performance was considerably better when fixation was unrestricted than when it was restricted. To our knowledge, these results

Table 1

Percentage Correct, $\mathbf{A}^{\prime}$, and Response Times

(Correct Responses Only, in Milliseconds) for the

Recognition Session as a Function of Learning Condition.

\begin{tabular}{lcccc}
\hline Recognition Condition & Hit Rate (\%) & False Alarm Rate (\%) & A $^{\prime}$ & RT \\
\hline After free viewing & 81.3 & & 0.91 & 2,166 \\
After restricted viewing & 52.5 & & 0.80 & 2,078 \\
New faces & & 11.9 & & 2,354 \\
\hline
\end{tabular}


provide the first experimental demonstration that eye movements facilitate face learning.

\section{Are the eye-movement patterns generated during face learning recapitulated during recognition?}

According to the scan path theory of perceptual recognition, eye movements generated during learning must be recapitulated during recognition. This theory predicts that when eye movements are prevented during learning, participants should refrain from making eye movements during recognition. A weaker prediction is that if eye movements are in fact generated during recognition following restricted viewing learning, such movements should differ from those generated during recognition following free viewing learning.

To examine the stronger prediction, we looked at the extent to which participants spontaneously made eye movements in the recognition session as a function of the learning condition. We quantified spontaneous eye movement in recognition as movement taking the eyes more than $1^{\circ}$ in any direction from the initial fixation position. Such trials were $84 \%$ in the free viewing condition and $85 \%$ in the restricted viewing condition $(F<1)$. Participants were as likely to move their eyes away from the initial fixation point in the recognition session following fixed viewing learning as following free viewing learning. This finding is not consistent with the strong prediction of the scan path theory.
Given that participants spontaneously moved their eyes equivalently in the recognition session regardless of whether they were allowed to do so in the learning session, the next question we asked was whether the eye movement patterns were similar in these two conditions. To examine this question, we compared the amount of time spent on the seven defined facial regions in the free viewing condition of the learning session with the amount of time spent on those regions in the test session as a function of learning condition. ${ }^{1}$ A direct comparison of viewing time on specific regions in the learning and recognition sessions is problematic because the faces were viewed for $10 \mathrm{sec}$ during the learning session but only about $2 \mathrm{sec}$ on average in the recognition session. To control for this difference in total viewing time but still allow comparison across conditions, fixation time in each region in each trial was calculated as a proportion of total fixation time for that trial. Figure 5 shows the proportion of viewing time as a function of viewing task and face region.

As can be seen in Figure 5, the proportion of time spent on each region was remarkably similar in the free viewing learning session and recognition session, as well as in the recognition session following the two learning conditions. Overall, the rank ordering of the proportion of fixation time spent over the features of the faces was similar across conditions, with the majority of time spent on the eyes, followed by the nose and then the mouth, with the other features receiving much less attention. To

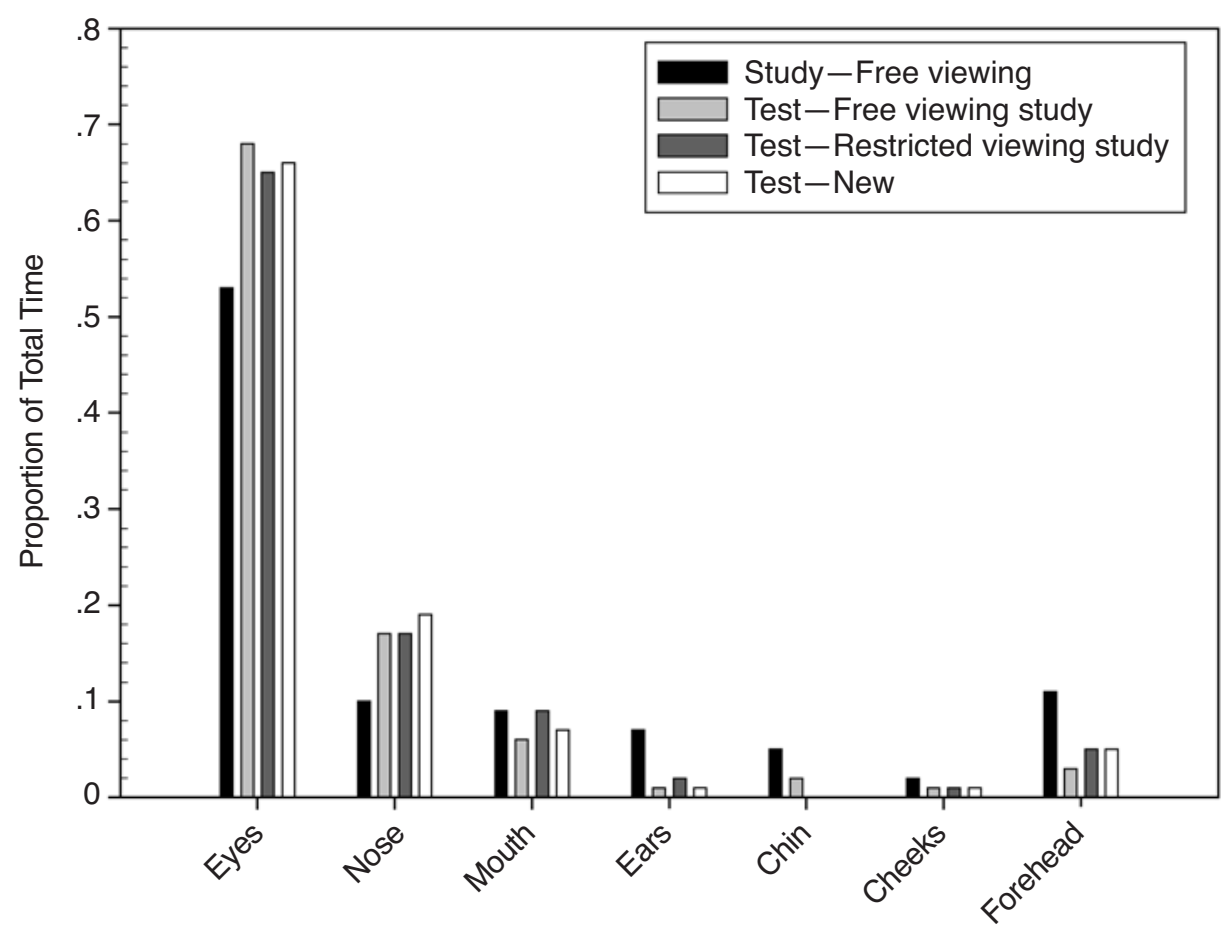

Figure 5. Mean proportion of total viewing time in each face region for the free viewing learning session and the recognition session. 
test the weak prediction of the scan path theory, we examined whether the proportion of time spent on each region during recognition differed as a function of learning condition. As can be seen in Figure 5, these proportions were extremely similar $(F<1)$. Thus, the weak form of the scan path theory was not supported by the data.

\section{Do eye movements change during face learning and recognition?}

It is clear from the pattern in Figure 5 that the distribution of fixation time across face features became more restricted from learning to recognition, with more time in the recognition session devoted to the features typically found to be more important in recognition (particularly the eyes and nose) and less time to the other features. This observation was supported by statistical analyses in which the proportion of fixation time in the free viewing learning session was compared with the average of the two recognition conditions. The proportion of total viewing time spent on the eyes and nose reliably increased, and the proportion of time spent on the ears, chin, and forehead reliably decreased, from learning to recognition [all $t \mathrm{~s}(7)>2.4$, all $p \mathrm{~s}<.05$ ]. The proportion of time spent viewing the cheeks decreased marginally $[t(7)=$ $2.12, p=.072]$, and the proportion of time on the mouth region was statistically equivalent in learning and recognition $[t(7)=.622, p>.30]$. These results are consistent with earlier findings that eye movements become more restricted during recognition than during learning (Althoff \& Cohen, 1999).

Finally, we asked whether the observed change in viewing time across features from learning to recognition was a function of the difference in prior exposure to the faces (a familiarity effect), or whether instead the change was due to the difference in the viewing task (a task effect). If the familiarity hypothesis is correct, the proportion of viewing time across features in the recognition session should differ for old and new faces, and viewing time for new faces in the recognition session should be similar to that observed in the learning session. If the task hypothesis is correct, the proportion of viewing time across features in the recognition session should be similar for old and new faces, and both should be different from that observed in the learning session. As can be seen in Figure 5, the data supported the latter hypothesis. In an analysis of the proportion of viewing time across regions in the learning session versus the new faces in the recognition session, there was a reliable interaction of condition $\times$ region $\left[F(6,42)=7.324, M S_{\mathrm{e}}=0.0032, p<.001\right]$. This interaction reflects the restriction in time spent across regions from learning to recognition, as was discussed above. A significantly smaller proportion of time was spent on the eyes and nose, and a significantly larger proportion of time was spent on the ears, chin, and forehead during the learning session than on the new faces in the recognition session (all $t \mathrm{~s}>2.4, p<.05$ ). No such differences were observed when comparing the old and new faces in the recognition session $(F<1)$. It appears that the restriction in feature sampling observed in face recognition compared with face learning was due not to familiarity, but rather to changes in feature selection for the tasks of learning and recognition.

\section{DISCUSSION}

This study was designed to determine whether eye movements play a functional role in face learning. The results were clear: Recognition accuracy was $28 \%$ higher when participants were allowed to move their eyes over the faces during learning than when they were required to hold fixation steady. This represents the first direct evidence that eye movements play a functional role in face learning.

If faces are processed holistically, what role might eye movements play in the learning process? One possibility is that initial encoding of relations among features benefits from foveal analysis. For example, determining the distance between the eyes in a face, or the relationship between (1) the distance between the eyes in a particular face and (2) the average distance between eyes in faces in general (i.e., second-order relations), may benefit from fixation on or near those features. A second possibility is that the eye movements themselves are functional in determining relations among features. For example, the length of a saccade between two eyes provides direct information about the distance between those eyes. A third possibility is that encoding of specific feature detail during learning is important for achieving a high level of recognition performance for faces that are not highly familiar. Additional studies will be required to tease these possibilities apart. In any case, it is clear that the information used to encode faces, whether holistic or not, is not derived from low-spatial frequency analysis taking place solely outside of foveal vision.

This study allowed us to examine two additional related questions. First, we examined the nature of the features selected for fixation during learning and recognition, and for learned and unlearned faces during recognition. In contrast to Yarbus (1967), but consistent with other eyemovement studies of face processing (e.g., Althoff \& Cohen, 1999; Henderson et al., 2001; Walker-Smith et al., 1977), we found that fixation was directed predominantly to internal facial features during recognition. The present study extended these results to face learning and to both old and new faces during recognition.

Second, consistent with Althoff and Cohen (1999), we found a greater concentration of fixation time on fewer critical features in recognition than in learning. However, contrary to the idea that this restriction in feature selection is due to prior exposure and therefore familiarity with the old faces, a similar concentration of time on the critical features was observed in the recognition session for both the previously learned and the new distractor faces. These results suggest that the concentration of feature selection is a task effect rather than a familiarity effect. 
Finally, how similar is feature selection during face learning and recognition? According to the scan path theory, the features selected for eye fixation during learning should be recapitulated during recognition (Noton \& Stark, 1971a, 1971b). This view predicts that the eyes should not move during recognition if they were prevented from moving during learning. A weaker prediction is that if the eyes do move during recognition following restricted viewing learning, the features selected by these movements should be dissimilar to those selected during recognition following free movement learning. Both of these predictions were refuted by the data. The eyes moved through the faces during recognition when eye movements were prevented during learning. Furthermore, the eyes moved to the same features for the same proportion of fixation time during recognition following restricted and free viewing learning. These results are consistent with other findings in the literature that challenge the scan path theory (Henderson, 2003; Rayner, 1998) and are consistent with the idea that face processing during both learning and recognition is supported by a common system with similar information-gathering needs.

\section{REFERENCES}

Althoff, R. R, \& Cohen, N. J. (1999). Eye-movement-based memory effect: A reprocessing effect in face perception. Journal of Experimental Psychology: Learning, Memory, \& Cognition, 25, 997-1010.

BRANT, S. A., \& STARK, L. W. (1997). Spontaneous eye movements during visual imagery reflect the content of the visual scene. Journal of Cognitive Neuroscience, 9, 27-38.

Buswell, G. T. (1935). How people look at pictures. Chicago: University of Chicago Press.

Crane, H. D. (1994). The Purkinje image eyetracker, image stabilization, and related forms of stimulus manipulation. In D. H. Kelley (Ed.), Visual science and engineering: Models and applications (pp. 15-89). New York: Marcel Dekker.

Cutting, J. E., Alliprandini, P. M. Z., \& Wang, R. F. (2000). Seeking one's heading through eye movements. Psychonomic Bulletin \& Review, 7, 490-498.

Dubois, S., Rossion, B., Schiltz, C., Bodart, J. M., Micehl, C., Bruyer, R., \& Crommelinck, M. (1999). Effect of familiarity on the processing of human faces. NeuroImage, 9, 278-289.

Ellis, H. D., Shepherd, J. W., \& Davies, G. M. (1979). Identification of familiar and unfamiliar faces from internal and external features: Some implications for theories of face recognition. Perception, $\mathbf{8}$, 431-439.

Farah, M. J., Wilson, K. D., Drain, M., \& TANAKa, J. N. (1995). What is "special" about face perception? Psychological Review, 105, 482498.

Gauthier, I., Tarr, M. J., Anderson, A. W., Skudlarski, P., \& Gore, J. C. (1999). Activation of the middle fusiform "face area" increases with expertise in recognizing novel objects. Nature Neuroscience, 2, 568-573.

Goren, C. C., SARTY, M., \& Wu, P. (1975). Visual following and pattern discrimination of face-like stimuli by newborn infants. Pediatrics, 56, 544-545.

Gorno Tempini, M. L., Price, C. J., Josephs, O., Vandenberghe, R., CAPPa, S. F., KAPUR, N., \& Frackowiak, R. S. J. (1998). The neural systems sustaining face and proper-name association. Brain, 121, 2130-2138.

Groner, R., Walder, F., \& Groner, M. (1984). Looking at faces: Local and global aspects of scanpaths. In A. G. Gale \& F. Johnson (Eds.), Theoretical and applied aspects of eye movement research (pp. 523-527). New York: Elsevier.

Hayhoe, M. M., Shrivastava, A., Mruczek, R., \& Pelz, J. B. (2003).
Visual memory and motor planning in a natural task. Journal of Vision, 3, 49-63.

HENDERSON, J. M. (2003). Human gaze control during real-world scene perception. Trends in Cognitive Sciences, 7, 498-504.

Henderson, J. M., FalK, R. J., Minut, S., Dyer, F. C., \& MahadeVAN, S. (2001). Gaze control for face learning and recognition in humans and machines. In T. Shipley \& P. Kellman (Eds.), From fragments to objects: Segmentation processes in vision (pp. 463-481). New York: Elsevier.

Henderson, J. M., \& Ferreira, F. (2004). Scene perception for psycholinguists. In J. M. Henderson \& F. Ferreira (Eds.), The integration of language, vision, and action: Eye movements and the visual world (pp. 1-58). New York: Psychology Press.

JaniK, S. W., Wellens, A. R., Goldberg, M. I., \& Dell'Osso, J. F. (1978). Eyes as the center of focus in the visual examination of human faces. Perceptual \& Motor Skills, 47, 857-858.

Kanwisher, N., McDermott, J., \& Chun, M. (1997). The fusiform face area: A module in human extrastriate cortex specialized for the perception of faces. Journal of Neuroscience, 17, 4302-4311.

KAnWISHeR, N., \& Moscovitch, M. (2000). The cognitive neuroscience of face processing: An introduction. Cognitive Neuropsychology, 17, 1-11.

Land, M., Mennie, N., \& Rusted, J. (1999). Eye movements and the roles of vision in activities of daily life: Making a cup of tea. Perception, 28, 1311-1328.

LURIA, S. M., \& STRAUSS, M. S. (1978). Comparison of eye movements over faces in photographic positives and negatives. Perception, $\mathbf{7}_{2}$ 349-358.

McCarthy, G., Puce, A., Gore, J. C., \& Allison, T. (1997). Facespecific processing in the human fusiform gyrus. Journal of Cognitive Neuroscience, 9, 605-610.

MerTens, I., SiegmuND, H., \& Grüsser, O. (1993). Gaze motor asymmetries in the perception of faces during a memory task. Neuropsychologia, 31, 989-998.

Minut, S., Mahadevan, S., Henderson, J. M., \& Dyer, F. (2000). Face recognition using foveal vision. In S.-W. Lee, H. H. Bulthoff, \& T. Poggio (Eds.), Biologically motivated computer vision. Berlin: Springer-Verlag.

Newcombe, F., Mehta, Z., \& de Haan, E. H. F. (1994). Category specificity in visual recognition. In M. J. Farah \& G. Ratcliff (Eds.), The neuropsychology of high-level vision (pp. 103-132). Hillsdale, NJ: Erlbaum.

Noton, D., \& Stark, L. (1971a). Scan paths in eye movements during pattern perception. Science, 171, 308-311.

Noton, D., \& STARK, L. (1971b). Scan paths in saccadic eye movements while viewing and recognizing patterns. Vision Research, 11, 929-942.

OLIVA, A., \& Schyns, P. G. (2000). Colored diagnostic blobs mediate scene recognition. Cognitive Psychology, 41, 176-210.

Potter, M. C. (1976). Short-term conceptual memory for pictures. Journal of Experimental Psychology: Human Learning \& Memory, 2, 509-522.

RAYNER, K. (1998). Eye movements in reading and information processing: 20 years of research. Psychological Bulletin, 124, 372422.

Schyns, P., \& Oliva, A. (1994). From blobs to boundary edges: Evidence for time- and spatial-scale-dependent scene recognition. Psychological Science, 5, 195-200.

STARK, L., \& ElLIS, S. R. (1981). Scanpath revisited: Cognitive models of direct active looking. In D. F. Fisher, R. A. Monty, \& J. W. Senders (Eds.), Eye movements: Cognition and visual perception. Hillsdale, NJ: Erlbaum.

TAnaka, J., \& Farah, M. (2003). The holistic representation of faces. In M. J. Peterson \& G. Rhodes (Eds.), Perception of faces, objects, and scenes: Analytic and holistic processes (pp. 53-74). New York: Oxford University Press.

Tanenhaus, M. K., Spivey-Knowlton, M. J., Eberhard, K. M., \& Sedivy, J. E. (1995). Integration of visual and linguistic information in spoken language comprehension. Science, 268, 1632-1634.

Thorpe, S. J., Fize, D., \& Marlot, C. (1996). Speed of processing in the human visual system. Nature, 381, 520-522. 
Tinker, M. A. (1946). The study of eye movements in reading. Psychological Bulletin, 43, 93-120.

Tippett, L. J., Miller, L. A., \& Farah, M. J. (2000). Prosopamnesia: A selective impairment in face learning. Cognitive Neuropsychology, 17, 241-255.

Walker-Smith, G. J., Gale, A. G., \& Findlay, J. M. (1977). Eye movement strategies in face perception. Perception, 6, 313-326.

YARBus, A. (1967). Eye movements and vision. New York: Plenum.

YIN, R. (1969). Looking at upside-down faces. Journal of Experimental Psychology, 81, 141-145.

Young, A. W., Hay, D. C., McWeeny, K. H., Flude, B. M., \& ELLIS, A. W. (1985). Matching familiar and unfamiliar faces on internal and external features. Perception, 14, 737-746.

\section{NOTE}

1. We also examined several other measures of the distribution of fixations over faces, including probability of entering each region and number of fixations in each region. The results from these analyses mirrored those from the fixation time analysis. We report the former because the derived proportion values for time are more easily generated and interpreted, and because total time tended to be slightly more sensitive to differences among conditions.

(Manuscript received April 7, 2003; revision accepted for publication April 23, 2004.) 